\title{
A PROOF OF SOME THEOREMS ON POINTWISE
}

\section{DISCONTINUOUS FUNCTIONS*}

\author{
BY \\ EDWARD B. VAN VLECK
}

BAIRE in his important thesis $\uparrow$ has given a number of interesting theorems concerning pointwise discontinuous functions. For their demonstration he employs the concept of semi- (i. e., upper or lower) continuity. $\ddagger$ However necessary the concept may be for subsequent portions of his investigation, its introduction is not needed for the particular theorems referred to. When the unnecessary element is removed, the principles of their demonstration, although very different in foim, become equivalent to those used by OsGOoD $\S$ in the derivation of his theorem relating to the convergence of series of continuous functions. (Cf. $\S 5$ below.)

The object of the following paper is, in part, to establish the theorems without going so far as to introduce the notion of upper continuity, thus reducing the proof to somewhat lower terms. It is found that BAIRE has restricted $f(x, y)$ more than is necessary in the following theorem :

If $f(x, y)$ is continuous in $x$ and $y$ separately and has a partial derivative $\partial f / \partial x$ at every point of

$$
T: \quad a \leqq x \leqq b, \quad c \leqq y \leqq d,
$$

this derivative, regarded as a function of $(x, y)$, is at most only pointwise discontinuous.

In fact, it suffices here to add to the hypothesis of the existence of $\partial f / \partial x$ the condition that $f(x, y)$ shall be continuous in $y$ upon a set of parallels to the $Y$-axis which are everywhere dense in $T$ (see theorem II). Other extensions of BAIRE's results will be noted further on (cf. theorems III and V).

It is believed that in the analysis given here the necessary principles have been arranged and combined in so simple a form as to permit quick and easy

\footnotetext{
* Presented to the Society October 29, 1904, with a supplementary note April 29, 1905. Received for publication January 16, 1907.

† Annali di Matematica, ser. 3, vol. 3 (1899).

$\ddagger$ Loc. cit., p. 6, or see Schönflizs' admirable Bericht über die Mengenlehre, Jahresberich t der deatsohen Mathematiker-Vereinigung, vol. 8 (1900), p. 141. Reference to this report will be indicated hereafter by the word Bericht.

\& A merican Journal of Mathematics, vol. 19 (1897), p. 155.
} 
application to other problems of like character which may arise. As instances of such application I will cite the two following theorems, which $I$ think are new and which constitute a second object of this paper :

Theorem VI.* If throughout the field

$$
T: \quad a \leqq x \leqq b, \quad c \leqq y \leqq d,
$$

the function $f(x, y)$ is continuous in $x$ when $y$ is constant and in $y$ when $x$ is constant, then

$$
F(x) \equiv \int_{c}^{d} f(x, y) d y
$$

is at most a pointwise discontinuous function in the interval $a \leqq x \leqq b$.

TheOREM VII. If at every point of $T$ the integrand possesses a partial derivative $f_{x}^{\prime}(x, y)$ which is continuous in the variables $x$ and $y$ considered separately, the integral $F(x)$ will have a derivative in some set of points which is everywhere dense in $(a, b)$, and the value of the derivative at the points here found will be obtained by differentiating with respect to the parameter under the integral sign; $i . e .$,

$$
F^{\prime}(x)=\int_{c}^{d} f_{x}^{\prime}(x, y) d y
$$

Furthermore, these points are points of continuity for

$$
\int_{c}^{d} f_{x}^{\prime}(x, y) d y
$$

Concerning the former of the two theorems it may be remarked that it has been known previously $\dagger$ that when $|f(x, y)|$ has an upper limit in $T$ the integral $F^{\prime}(x)$ is continuous in $(a b)$. The cases of pointwise discontinuity therefore arise only when there is no upper limit for $|f(x, y)|$.

\section{$\S 1$. The preliminary principles.}

We shall first demonstrate the following very simple

Fundamental Principle : $\ddagger$ If to every point $p_{i}$ of a continuum (or of a perfect set of points) $\S$ a positive number $\delta_{i}$ is assigned in any manner, then there is some portion of the continuum (some perfect subset) where the points $q_{i}$

* The two theorems are numbered with reference to the place in which they are proved later on.

† Arzera, Atti della R. Accademia dei Lincei, ser. 4, vol. 1 (1884-5), p. 537. It also can be deduced immediately from OsGoov's theorem 5, loo. cit., p. 182.

† A special case in which this principle is used incidentally will be found in the work of OsGood, Mathematische Annalen, vol. 53 (1900), p. 463 . Other instances of the use of the principle can doubtless be fonnd.

\& No restriction is here placed upon the perfect point-set. It may consist of a set of points nowhere dense in the space in which it is contained. The continuum may be either closed or open. 
which have $\delta_{i}>D$ are everywhere dense (dense with respect to the subset), ${ }^{*}$ provided $D$ is taken sufficiently small.

Let us suppose, if possible, that this is not true. Denote by $\zeta_{1}, \zeta_{2}, \zeta_{3}, \ldots$ any sequence of decreasing positive numbers having 0 as their limit. Further let $Q_{i}$ denote the set of points $q_{i}$ for which $\delta_{i}>\zeta_{i}$. Clearly $Q_{i}$ will be contained in $Q_{i+1}$. If, now, the principle stated is false, then there is some portion of the continuum (some perfect subset) which contains no points of $Q_{1}$. Within this portion (subset) there must be another part (perfect set) which is free from the points of $Q_{2}$, and so on. The limit of this series of sub-portions (sub-sets), contained each within the preceding, is either a point $\dagger$ to which belongs a non-negative number smaller than any $\zeta_{i}$, or a set of such points. But 0 is the only number having this property. This, however, contradicts the hypothesis that to every point there corresponds a positive number. The fundamental principle is therefore established.

By placing $\delta_{i}=1 / \delta_{i}^{\prime}$ and $D=1 / D^{\prime}$ the principle may be put also into the following form: If to every point of the continuum a positive number $\delta_{i}^{\prime}$ is assigned in any way, the points $q_{i}$ for which $\delta_{i}^{\prime}<D^{\prime}$ must be everywhere dense in some portion of the continuum, provided $D^{\prime}$ is sufficiently large.

Consider next the set $S$ of points $s_{i}$ for which the assigned numbers $\delta_{i}$ fall between $D$ and $1 / D$. It will be seen at once that there must be some portion of the continuum (some perfect subset) in which the points $s_{i}$ are everywhere dense, provided, of course, that $D$ is sufficiently small. Let the interval $(D, 1 / D)$ be divided into any number of equal parts,

$$
\epsilon=\frac{1 / D-D}{n},
$$

and denote by $S_{1}, S_{2}, \cdots, S_{n}$ the subsets of $S$ for which the values of $\delta_{i}$ fall successively in the intervals $(D, D+\epsilon),(D+\epsilon, D+2 \epsilon), \cdots,(1 / D-\epsilon, 1 / D)$. Since the points of $S$ are everywhere dense in the part of the continuum (perfect set) now under consideration, there must be some subportion (some perfect subset) in which the points of some one of the sets $S_{i}$ are everywhere dense. As, moreover, the value of $\epsilon$ may be made as small as desired, we obtain the following simple

Extension of The FUNDAMENTAL PRINCIPLE: If to every point of a continuum (perfect set of points) a positive number $\delta_{i}$ is assigned in any manner, then for a sufficiently small value of $D$ there is some portion of the continuum (some perfect subset) containing a set of points everywhere dense, for which the values of $\delta_{i}$ not only are greater than $D$ but differ from one another by less than an arbitrarily assigned positive quantity $\epsilon$.

\footnotetext{
* The points $q_{i}$ are said to be dense with respect to the subset, if within a sphere of any radius, described about any point of the subset as center, there lies always a point $q_{i}$.

†C. Bericht, p. 58.
} 
In the case of a continuum of two or more dimensions it will be desirable not merely to know the existence of some portion of the continuum in which the points $q_{i}$ are everywhere dense, but to ascertain also their distribution on a field of curves or surfaces which completely fill that portion. Inasmuch as every surface can be regarded as composed of curves, it will suffice here to consider the distribution of the $q_{i}$ with respect to a continuous curve-system. For simplicity we shall confine our attention to a system of parallel straight lines, the results obtained being easily capable of extension to other curve-systems.

Let first a portion of space be selected from the continuum in the form of a right cylinder having its axis parallel to the given system of parallel straight lines. For the case of a two dimensional continuum the cylinder shall be understood to be a rectangle. Consider then the segment intercepted upon any one of the parallels by the cylinder. Since each segment is itself a continuum, there must be for a sufficiently small value of $D$ some portion of the segment in which the points $q_{i}$ having $\delta_{i}>D$ are everywhere dense. Denote for any segment the length of such a portion by $l_{i}$ and the corresponding value of $D$ by $D_{i}$. We will then assign to the segment (or, if we prefer, to its intersection with the base of the cylinder) the smaller of the two numbers $l_{i}$ and $D_{i}$. Call it $s_{i}$. Now the set of segments (intersections) is perfect, and therefore by our "fundamental principle" the segments (intersections) for which $s_{i}>S$ must be everywhere dense in some portion of the cylinder (base), provided that $S$ is a sufficiently small number. Take any such portion and let this dense set of segments be denoted by $G$. Divide any element of this new portion into equal parts not exceeding $S / 2$ in length, and through the points of division pass planes parallel to the base of the cylinder, thereby dividing the portion under consideration into a finite number of component cylinders. Each segment of $G$ contains at least one interval of length $s_{i}>S$ along which the points $q_{i}$ having $\delta_{i}>s_{i}>S$ are everywhere dense. Consequently some one of the component cylinders must intercept an interval of length $S / 2$, upon which the points $q_{i}$ are everywhere dense. Since also the lines of $G$ were everywhere dense before the subdivision into component cylinders took place, these intercepted intervals of length $S / 2$ must be everywhere dense in some portion of one or more of the component cylinders. We obtain thus the following addendum to our fundamental principle:

AdDendum. When the continuum is a portion of a plane or a space of higher dimensions, it is possible to select the region in which the points $q_{i}$ are everywhere dense so as to fulfill the following condition: For a sufficiently small but fixed value of $D$ there is throughout the region a dense set of parallels, upon each of which the $q_{i}$ are everywhere dense; and the direction of the parallels may be assigned arbitrarily before seeking the region. 


\section{§ 2. Application to derivatives.}

Let $f(x)$ be a function of a real variable having a derivative at every point of an interval $(a, b)$ of the $X$-axis. If $\epsilon$ be a small arbitrarily assigned positive quantity, any point $p_{i}$ in $(a, b)$ can be made the center of an interval $2 \delta_{i}$ within which

$$
\left|\frac{f\left(p_{i}+h\right)-f\left(p_{i}\right)}{h}-f^{\prime}\left(p_{i}\right)\right|<\epsilon \quad\left(|h|<\delta_{i}\right) .
$$

I shall assign to each point $p_{i}$ such a positive number $\delta_{i}$. Then, by $\S 1$, for a sufficiently small $D$ the points $q_{i}$ for which $\delta_{i}>D$ must be dense in some portion of $(a, b)$. Let $\left(a^{\prime}, b^{\prime}\right)$ be such a portion, and denote by $Q_{i}$ the dense set of points $q_{i}$ within $\left(a^{\prime}, b^{\prime}\right)$. Then for any two points $q_{1}$ and $q_{2}$ whose distance $\left|q_{1}-q_{2}\right|$ does not exceed $D$ we have

$$
\left|\frac{f\left(q_{1}\right)-f\left(q_{2}\right)}{q_{1}-q_{2}}-f^{\prime}\left(q_{1}\right)\right|<\epsilon, \quad\left|\frac{f\left(q_{1}\right)-f\left(q_{2}\right)}{q_{1}-q_{2}}-f^{\prime}\left(q_{2}\right)\right|<\epsilon,
$$

and therefore

$$
\left|f^{\prime}\left(q_{1}\right)-f^{\prime}\left(q_{2}\right)\right|<2 \epsilon .
$$

Consequently the variation of $f^{\prime}(x)$, taken for the set $Q_{i}$ and for any subinterval of $\left(a^{\prime}, b^{\prime}\right)$ not greater than $D$ in length, will not be greater than $4 \epsilon$.

The fundamental principle has been used thus to obtain a portion of the continuum in which there is a dense set of points with a certain specified property. Our second step in this and subsequent demonstrations consists in the extension of the property to all points of the portion. In the special case before us now, every point $p$ of $\left(a^{\prime}, b^{\prime}\right)$, and in particular a point $p$ which does not beiong to $Q_{i}$, has a small neighborhood $2 h$ such that

$$
\left|\frac{f\left(q_{i}\right)-f(p)}{q_{i}-p}-f^{\prime}(p)\right|<\epsilon^{\prime} \quad\left(\left|q_{i}-p\right|<h\right),
$$

where $\epsilon^{\prime}$ is an arbitrary small prescribed quantity. But also when $\left|q_{i}-p\right| \leqq D$, we have

Consequently

$$
\left|\frac{f\left(q_{i}\right)-f(p)}{q_{i}-p}-f^{\prime}\left(q_{i}\right)\right|<\epsilon .
$$

$$
\left|f^{\prime}(p)-f^{\prime}\left(q_{i}\right)\right|<\epsilon+\epsilon^{\prime} .
$$

As $\epsilon^{\prime}$ is arbitrarily small, we conclude from (1) and (2) that the variation of $f^{\prime}(x)$ in any portion of $\left(a^{\prime}, b^{\prime}\right)$ not exceeding $D$ in length will not exceed $\zeta_{1}=6 \epsilon$.

This brings us to the third and final step of this and similar demonstrations; namely, the establishment of the pointwise discontinuous character of the function under consideration after it has been demonstrated that there is some por- 
tion of the original field in which the variation of the function does not exceed an arbitrarily prescribed quantity $\zeta_{1}$. The reasoning is so familiar that $I$ give it here once for all, remarking merely that it is applicable to fields of two or more dimensions as well as to intervals. Take any series of decreasing positive numbers $\zeta_{1}, \zeta_{2}, \ldots$ having 0 as their limit. Inside the part of the field in which the variation does not exceed $\zeta_{1}$ there must be, by like reasoning, a part in which the variation does not exceed $\zeta_{2}$; in the interior of this another part in which the variation does not exceed $\zeta_{3}$, and so on. The limit of this series of intervals or regions, taken each entirely in the interior of the preceding, is a point (or set of points) at which the function is continuous. Furthermore, as the argument applies to any portion of the initial field, the points of continuity must be everywhere dense, and the function is at most only pointwise discontinuous.

Applied to $f^{\prime}(x)$ in the particular case before us, this reasoning gives at once the following theorem :

Theorem I. If a function of a real variable $x$ has a derivative at every point of an interval $(a, b)$, this derivative is at most only pointwise discortinuous in the interval (BAIRE).*

It is well known that the points of continuity in the case of a pointwise discontinuous function have the power of a continuum. The same remark applies to all other sets of points obtained in this paper (cf. theorem VII) by the above method. In fact, to each value of $\zeta_{i}$ there was found between $a$ and $b$, or between any two points of $(a, b)$, a certain interval with specified property. Consequently the totality of points which cannot be included in the interior of such intervals is nowhere dense in $(a, b)$. Call this set of points $B_{i}$. Corresponding to $\zeta_{1}, \zeta_{2}, \ldots$ we have an enumerable set of such sets $B_{i}$, each nowhere dense in $(a, b)$, and these together constitute what BAIRE calls a point-set of the first category. $\dagger$ The complementary point-set, which consists of such points as we obtain by our method, has the power of a continuum. $\ddagger$

Theorem I may be extended to one-side derivatives. The extension is not without some interest, for it is known that the four derivatives of a function, when distinct throughout an interval, must be totally discontinuous. § The possibilities in respect to continuity are accordingly settled for the various species of derivatives. For extension of the above theorem to one-side derivatives BaIRE's proof requires no modification. The proof given here may be modified as follows.

Suppose that only a right-hand derivative $f_{+}^{\prime}(x)$ exists in $(a, b)$. Assign

* BAIRE deduces the theorem as a corollary of the fundamental theorem which will be quoted at the beginning of $z 3$; loc. cit., p. 64 or Bericht, p. 224 .

$\dagger$ Loo. cit., p. 65.

$\ddagger$ Cf. Bericht, p. 108.

¿ Cf. DinI's Grundlugen für die T'heorie der Functionen einer reellen Grösse, p. 249. 
to each point $p_{i}$ of the interval a positive number $\delta_{i}$ such that

$$
\left|\frac{f\left(p_{i}+h\right)-f\left(p_{i}\right)}{h}-f_{+}^{\prime}\left(p_{i}\right)\right|<\frac{\epsilon}{4} \quad\left(0<h<\delta_{i}\right) .
$$

For a sufficiently small $D$ there will be some subinterval $\left(a^{\prime}, b^{\prime}\right)$ in which the points $q_{i}$ for which $\delta_{i}>D$ are everywhere dense. Take within it two such points, $q_{1}$ and $q_{2}=q_{1}+h$, for which $0<h \leqq D / 2$. We have the three inequalities

$$
\begin{gathered}
\left|\frac{f\left(q_{1}+h\right)-f\left(q_{1}\right)}{h}-f_{+}^{\prime}\left(q_{1}\right)\right|<\frac{\epsilon}{4}, \\
\left|\frac{f\left(q_{1}+2 h\right)-f\left(q_{1}+h\right)}{h}-f_{+}^{\prime}\left(q_{1}+h\right)\right|<\frac{\epsilon}{4}, \\
\left|\frac{f\left(q_{1}+2 h\right)-f\left(q_{1}\right)}{2 h}-f_{+}^{\prime}\left(q_{1}\right)\right|<\frac{\dot{\epsilon}}{4},
\end{gathered}
$$

which combined together give

or

$$
\left|f_{+}^{\prime}\left(q_{1}+h\right)+f_{+}^{\prime}\left(q_{1}\right)-2 f_{+}^{\prime}\left(q_{1}\right)\right|<\epsilon
$$

$$
\left|f_{+}^{\prime}\left(q_{2}\right)-f_{+}^{\prime}\left(q_{1}\right)\right|<\epsilon .
$$

Consequently for $Q_{i}$-, i. e., the set of points $q_{i}$ within $\left(a^{\prime}, b^{\prime}\right)$ - the variation of $f_{+}^{\prime}(x)$ will not exceed $2 \epsilon$ in any segment not exceeding $D / 2$ in length.

Take next any point $p$ of $\left(a^{\prime}, b^{\prime}\right)$ not belonging to $Q_{i}$ and let $\delta<D$ be a number assigned to it in accordance with (3). Since $p$ is the limit of a set of points $q_{i}$ which lie to the right of it, we may choose from $Q_{i}$ some point $q_{i}=p+h$ for which $0<h<\delta / 2$. Then if we replace $q_{1}$ by $p$ and $q_{2}$ by $q_{i}$, the inequalities (4)-(7) will hold as before. Consequently

$$
\left|f_{+}^{\prime}\left(q_{i}\right)-f_{+}^{\prime}(p)\right|<\epsilon .
$$

It follows therefore that the variation of $f_{+}^{\prime}(x)$ for any portion of $\left(a^{\prime}, b^{\prime}\right)$ which does not exceed $D / 2$ in length will not be greater than $\zeta_{1}=4 \epsilon$. The conclusion that $f_{+}^{\prime}(x)$ is at most only pointwise discontinuous then follows in the manner previously described.

We shall consider next a function $f(x, y)$ which has a partial derivative $\partial f / \partial x$ at every point of a field $T$ defined by

$$
a \leqq x \leqq b, \quad c \leqq y \leqq d .
$$

By virtue of this hypothesis $f(x, y)$ must be continuous with respect to $x$. We suppose also that it is continuous with respect to $y$ upon a set of parallels $x=x_{i}$ to the $Y$-axis which are everywhere dense in $T$, and proceed then to examine the character of $\partial f / \partial x$ in regard to continuity when both $x$ and $y$ are 
varied. Any point $p=(x, y)$ may be made the center of an interval parallel to the axis of $x$ and having such a length $* 2 \delta$ that

$$
\left|\frac{f(x+h, y)-f(x, y)}{h}-\frac{\partial f(x, y)}{\partial x}\right|<\epsilon \quad(|h|<\delta) .
$$

By the "addendum" of $\S 1$ it is possible for a sufticiently small value of $D$ to find a part of $T$

$$
T^{\prime}: \quad a^{\prime} \leqq x \leqq b^{\prime}, \quad c^{\prime} \leqq y \leqq d^{\prime}
$$

where the points $q_{i}$ having $\delta_{i}>D$ will be everywhere dense upon each line of some system of parallels, $y=y_{j}$, which is itself everywhere dense throughout $T^{\prime}$. Let $x=x_{1}$ and $x=x_{1}+h$ be any two lines within $T^{\prime}$ along which $f(x, y)$ is continuous with respect to $y$. Then, $h$ being fixed in value, the difference quotient

$$
r\left(x_{1}, y\right)=\frac{f\left(x_{1}+h, y\right)-f\left(x_{1}, y\right)}{h}
$$

will be a continuous function of $y$ on the line $x=x_{1}$. Denote by $L L^{\prime}$ any segment of the line in which the variation of $(9)$ is less than a prescribed $\epsilon$, and upon $L L^{\prime}$ as a side construct within $T^{\prime}$ a rectangle $L L^{\prime} M^{\prime} M$ having its second side $L^{\prime} M^{\prime}<D$.

Consider the variation of $\partial f(x, y) / \partial x$ in this rectangle, and first of all upon the dense set of parallels $y=y_{j}$. If the intersection $\left(x_{1}, y_{j}\right)$ of $x_{1}$ with one of these parallels is not itself one of the points $q_{i}$, it is the limit of a set of such points upon $y=y_{j}$, and we may take a point $q_{j}=\left(\bar{x}, y_{j}\right)$ so close to $\left(x_{1}, y_{j}\right)$ that the difference of the values of the different quotient $r(x, y)$ at these two points will be numerically less than an arbitrarily assigned quantity $\epsilon^{\prime}$. At the second point $q_{j}$ we have

$$
\left|r\left(\bar{x}, y_{j}\right)-\frac{\partial f\left(\bar{x}, y_{j}\right)}{\partial x}\right|<\epsilon
$$

Since, furthermore, on the one hand, the variation of $r(x, y)$ upon $L L^{\prime}$ is less than $\epsilon$, and, on the other hand, the variation of $\partial f / \partial x$ upon $y=y_{j}$ is less than $\zeta=6 \epsilon$ by the proof of theorem I, it follows at once that the total variation of $\partial f / \partial x$, taken for the set of parallels $y=y_{j}$, will be less than $\epsilon+2(6 \epsilon)+2\left(\epsilon+\epsilon^{\prime}\right)$. Since also $\epsilon^{\prime}$ is arbitrary, it will not exceed $15 \epsilon$.

Bring next into consideration the points of the rectangle which do not lie upon the dense set of parallels $y=y_{j}$. For any such point $\left(x^{\prime}, y^{\prime}\right)$ we can find another point $\left(x^{\prime \prime}, y^{\prime}\right)$ so near to it that

$$
\left|\frac{f\left(x^{\prime \prime}, y^{\prime}\right)-f\left(x^{\prime}, y^{\prime}\right)}{x^{\prime \prime}-x^{\prime}}-\frac{\partial f\left(x^{\prime}, y^{\prime}\right)}{\partial x}\right|<\epsilon^{\prime} .
$$

* In this and other cases BAIRE considers the maximum interval $2 \delta$ fulfilling the condition imposed and proves that $\delta$ as a funotion of $(x, y)$ is "semi-continue superieurement." In the method which is here being followed, it is unnecessary to introdnce the maximum $\delta$. 
Now if neither point lies upon a line of continuity for $f(x, y)$ with respect to $y$, we can take a pair of such points, $\left(\bar{x}^{\prime}, y^{\prime}\right)$ and $\left(\bar{x}^{\prime \prime}, y^{\prime}\right)$, so close to them that

$$
\left|\frac{f\left(\bar{x}^{\prime \prime}, y^{\prime}\right)-f\left(\bar{x}^{\prime}, y^{\prime}\right)}{\bar{x}^{\prime \prime}-\bar{x}^{\prime}}-\frac{f\left(x^{\prime \prime}, y^{\prime}\right)-f\left(x^{\prime}, y^{\prime}\right)}{x^{\prime \prime}-x^{\prime}}\right|<\epsilon^{\prime},
$$

while if either point lies upon such a line of continuity we have merely to identify it in (11) with the corresponding point of the second pair. Having now two points upon lines of $y$-continuity, by varying $y$ we can find one of the dense set of parallels $y=y_{j}$ so near to $y=y^{\prime}$ that

$$
\left|\frac{f\left(\bar{x}^{\prime \prime}, y_{j}\right)-f\left(\bar{x}^{\prime}, y_{j}\right)}{\bar{x}^{\prime \prime}-\bar{x}^{\prime}}-\frac{f\left(\bar{x}^{\prime \prime}, y^{\prime}\right)-f\left(\bar{x}^{\prime}, y^{\prime}\right)}{\bar{x}^{\prime \prime}-\bar{x}^{\prime}}\right|<\epsilon .
$$

But upon this parallel it is possible to select from the dense set of points $q_{i}$ such a point $q_{i}=\left(x_{i}, y_{i}\right)$ that

$$
\left|\frac{f\left(x_{i}, y_{j}\right)-f\left(\bar{x}^{\prime}, y_{j}\right)}{x_{i}-\bar{x}^{\prime}}-\frac{f\left(\bar{x}^{\prime \prime}, y_{j}\right)-f\left(\bar{x}^{\prime}, y_{j}\right)}{\bar{x}^{\prime \prime}-\bar{x}^{\prime}}\right|<\epsilon^{\prime} \quad\left(\left|x_{i}-\bar{x}^{\prime}\right|<D\right) .
$$

Since, lastly, for $q_{i}$

$$
\left|\frac{\partial f\left(x_{i}, y_{j}\right)}{\partial x_{i}}-\frac{f\left(x_{i}, y_{j}\right)-f\left(\bar{x}^{\prime}, y_{j}\right)}{x_{i}-\bar{x}^{\prime}}\right|<\epsilon,
$$

it follows immediately from (10)-(14) that

$$
\left|\frac{\partial f\left(x_{i}, y_{j}\right)}{\partial x}-\frac{\partial f\left(x^{\prime}, y^{\prime}\right)}{\partial x}\right|<\epsilon+4 \epsilon^{\prime},
$$

where $\epsilon^{\prime}$ is arbitrarily small. As the variation of $\partial f / \partial x$ upon the dense set of parallels $y=y_{j}$ does not exceed $15 \epsilon$, we conclude from the last inequality that the variation of $\partial f / \partial x$ in the rectangle $L L^{\prime} M^{\prime} N$ will not exceed $15 \epsilon+2 \epsilon=17 \epsilon$.

This rectangle can now be substituted in place of the original field. By indefinitely decreasing $\zeta_{1}=17 \epsilon$ we obtain as the limit of a series of rectangles, lying each within the preceding, a point of continuity of $\partial f / \partial x$ considered as a function of $(x, y)$. This proves the following theorem ;

TheOREM II. If $f(x, y)$ at every point of $T$ possesses a partial derivative $\partial f(x, y) / \partial x$ and if, furthermore, there is in $T$ an everywhere dense set of parallels $x=x_{i}$ upon which $f(x, y)$ is continuous in $y$, then $\partial f(x, y) / \partial x$ as a function of $(x, y)$ is at most only pointwise discontinuous.

For the sake of completeness I call attention to BAIRE's important remark* that when the derivative $\partial f / \partial y$ exists as well as $\partial f / \partial x$, there must be at least a set of points everywhere dense throughout $T$ at which both derivatives are continuous, and at which, therefore, $f(x, y)$ has a differential. The proof follows immediately by making the values of $\zeta_{1}, \zeta_{2}, \zeta_{3}, \ldots$ refer alternately to the variation of the two derivatives.

\footnotetext{
*Loc. cit. , pp. 108-110.
} 


\section{§3. Theorems on the continuity of $f(x, y)$.}

BAIRE in his thesis has given the following important theorem :

If $f(x, y)$ is continuous with respect to $y$ at every point of a rectangle

$$
T: \quad a \leqq x \leqq b, \quad c \leqq y \leqq d,
$$

and with respect to $x$ on a set of parallels to the $X$-axis everywhere dense in $T$, then $f(x, y)$ as a function of the two variables together is at most only point-wise discontinuous; and the points of continuity in $T$ will be everywhere dense upon any curve having an equation of the form $y=\phi(x)$, wherein $\phi(x)$ is a continuous function of $x . *$

SCHÖNFLIEs, $\dagger$ commenting on this theorem, has remarked that although BAIRE's hypothesis of continuity with respect to $x$ is stated for a dense set of parallel straight lines, this is clearly an unessential specialization, for in place of this set of parallels "eine überall dichte Curvenschar" could be substituted. There is, however, nothing in BAIRE's work to indicate the mode of distribution of the points of continuity along such a system of curves $y=\phi_{i}(x)$ as ScHönflies suggests. I shall complete the result by establishing the following theorem :

TheORem III. If $f(x, y)$ is continuous with respect to $y$ at every point of $T$ and with respect to $x$ upon a set of curves everywh : dense and having equations of the form $y=\phi_{i}(x)$, in which $\phi_{i}(x)$ is cous in $x$, then $f(x, y)$ as a function of $(x, y)$ is continuous at all the intersections of these curves with some set of parallels to the $Y$-axis which are likewise everywhere dense in $T$.

Proof. To every line $x=x_{i}$ in the field $T$ let a positive number $\delta_{i}$ be so assigned that the variation of $f^{\prime}(x, y)$ in any segment not exceedin $\delta_{i}$ in length will not exceed a fixed but arbitrarily prescribed $\epsilon$. According to ur fundamental principle, for a sufficiently small value of $D$ the set of lines $n=\xi_{i}$ for which $\delta_{i}>D$ must be dense in some portion of $T$. Suppose such a portion $T^{\prime}$ to be included between $x=\xi_{1}$ and $x=\xi_{2}$, and denote the dense set of lines between these limits by $\Xi$. We shall establish first that the characteristic property of the lines of $\Xi$ holds within $T^{\prime}$ for every parallel to the $Y$-axis.

Consider for this purpose any line $x=x^{\prime}$ which is not known to belong to Z. It is cut by the curves $y=\phi_{i}(x)$ in a dense set of points $\left(x^{\prime}, y_{i}\right)$. Take now any two points whatsoever of the line, $\left(x^{\prime}, y^{\prime}\right)$ and $\left(x^{\prime}, y^{\prime \prime}\right)$, for which $\left|y^{\prime}-y^{\prime \prime}\right| \leqq D$. If these points are not themselves included in the dense set of intersections $\left(x^{\prime}, y_{i}\right)$, we can take two of the intersections $\left(x^{\prime}, y_{i}\right),\left(x^{\prime}, y_{j}\right)$ which lie so near to these two points that simultaneously

$$
\begin{gathered}
\left|y_{i}-y_{j}\right| \leqq D, \\
\left|\left[f\left(x^{\prime}, y^{\prime}\right)-f\left(x^{\prime}, y^{\prime \prime}\right)\right]-\left[f\left(x^{\prime}, y_{i}\right)-f\left(x^{\prime}, y_{j}\right)\right]\right|<\epsilon^{\prime},
\end{gathered}
$$

* Loc. cit., p. 27.

$\dagger$ Bericht, p. 140. The specification as to their distribution upon $y=\phi(x)$ is omitted in ScHöNFLIKS' summary of the theorem (p. 142). 
in which $\epsilon^{\prime}$ is arbitrarily small. Then since $\left(x^{\prime}, y_{i}\right),\left(x^{\prime}, y_{j}\right)$ are points of continuity of $f(x, y)$ as a function of $x$, they may be made the centers of intervals parallel to the $X$-axis and so small that in either interval the variation of $f(x, y)$ will be less than $\epsilon^{\prime}$. Furthermore, since the lines of $\Xi$ are everywhere dense in $T^{\prime}$, the two intervals will be cut by certain lines of the set. Let $\left(\xi^{\prime}, y_{i}\right),\left(\xi^{\prime}, y_{j}\right)$ be the intersections of the two intervals with some one of these lines. We now have the inequalities

$$
\begin{gathered}
\mid\left[\left(f\left(x^{\prime}, y_{i}\right)-f\left(x^{\prime}, y_{j}\right)\right]-\left[f\left(\xi^{\prime}, y_{i}\right)-f\left(\xi^{\prime}, y_{j}\right)\right] \mid<2 \epsilon^{\prime},\right. \\
\left|f\left(\xi^{\prime}, y_{i}\right)-f\left(\xi^{\prime}, y_{j}\right)\right| \leqq \epsilon .
\end{gathered}
$$

It follows then by combination of these with the inequality above that

$$
\left|f\left(x^{\prime}, y^{\prime}\right)-f\left(x^{\prime}, y^{\prime \prime}\right)\right|<\epsilon+3 \varepsilon^{\prime},
$$

and hence, since $\epsilon^{\prime}$ is arbitrary,

$$
\left|f\left(x^{\prime}, y^{\prime}\right)-f\left(x^{\prime}, y^{\prime \prime}\right)\right| \leqq \epsilon .
$$

We have thus shown that the variation of $f(x, y)$ in $T^{\prime}$ upon any parallel to the $Y$-axis will not exceed $\epsilon$ for any segment of the parallel not greater than $D$ in length.

Any point $\left(x_{1}, y_{1}\right)$ within $T^{\prime}$ at which $f(x, y)$ is continuous with respect to $x$ can now be made the center of a rectangle, with sides parallel to the axes of $x$ and of $y$, in which the variation of $f(x, y)$ does not exceed $3 \epsilon$. For suppose it first to be made the center of an interval $\left(x_{1}-\delta^{\prime}, x_{1}+\delta^{\prime}\right)$ parallel to the $X$-axis in which the variation does not exceed $\epsilon$. Then the rectangle bounded by

$$
x=x_{1}-\delta^{\prime}, \quad x=x_{1}+\delta^{\prime}, \quad y=y_{1}-D, \quad y=y_{1}+D .
$$

or so much of it as lies in $T^{\prime}$, fulfills the conditions stated.

Let the value of $\epsilon$ be now diminished. Corresponding to an indefinitely diminishing series $\epsilon^{\prime}, \epsilon^{\prime \prime}, \epsilon^{\prime \prime \prime}, \ldots$ we will have a series of rectangles $T^{\prime}, T^{\prime \prime}, T^{\prime \prime \prime}, \ldots$ having the same altitude as $T$ and lying each within the preceding. The limit of this series of rectangles is a line $y=\eta$ (or a continuous set of such lines) which has the following property: Every point of the line at which $f(x, y)$ is continuous with respect to $x$ is also a point of continuity of $f(x, y)$ with respect to $(x, y)$, inasmuch as it can be enclosed in a series of rectangles in which the variation of $f(x, y)$ is successively not greater than $3 \epsilon^{\prime}, 3 \epsilon^{\prime \prime}, 3 \epsilon^{\prime \prime \prime}, \ldots$. In particular, every point in which the line is cut by the curves $y=\phi_{i}(x)$ is a point of continuity of $f(x, y)$.

If the original field $T$ is divided into two parts by a parallel to the $Y$-axis, our reasoning applies to each part. The lines $y=\eta$ are therefore everywhere dense in $T$. This proves the theorem. 
One particular case of theorem III is worthy of special note, that in which $f(x, y)$ is continuous in $x$ and $y$ separately at every point of $T$. Applied to this case the theorem becomes the following corollary :

Theorem IV. If $f(x, y)$ is continuous in $x$ and $y$ separately, there exists. in $T$ an everywhere dense set of parallels to the $Y$-axis (also to the $X$-axis), at every point of which $f(x, y)$ is continuous in $(x, y)$.

This is merely another statement of the following conclusion of BAIRE :*

TheоReм IV'. If $f(x, y)$ is continuous in $x$ and $y$ separately within $T$, the projections upon the axis of $x$ of all points of discontinuity whose measure is greater than an arbitrarily prescribed positive quantity $\sigma$ will be nowhere dense.

To establish the equivalence of the two statements, it suffices to note that, by the proof of IV, between any two parallels to the $Y$-axis containing points of discontinuity which have a measure greater than $\sigma$, there may be inserted one of our strips $T^{\prime}$ for which $\sigma_{1} \equiv 3 \epsilon$ may be taken less than $\sigma$. Because $3 \epsilon$ is an upper limit for the measure of discontinuity at all interior points of $T^{\prime}$, it follows that the projections of the discontinuities having a measure $>\sigma$ can be nowhere dense. Conversely, if $\mathrm{IV}^{\prime}$ be assumed, theorem IV at once results by application of familiar principles.

In the method which I have given here, attention has been concentrated upon the parallels of continuity for $f(x, y)$ rather than upon those containing points of discontinuity. This makes it easy to make the following advance beyond BAIRE's result :

Theorem V. Upon the hypothesis enunciated in theorem IV there exists also a set of lines everywhere dense in $T$ and parallel to the $Y$-axis, upon each of which $f(x, y)$ as a function of $(x, y)$ is uniformly continuous. $\dagger$

To prove this theorem consider again the strip $T^{\prime}$. Divide any interior line $x=x^{\prime}$ into segments equal to or less than $D / 2$ in length by means of the points

$$
\left(x^{\prime}, y_{1}\right),\left(x^{\prime}, y_{2}\right), \cdots,\left(x^{\prime}, y_{n}\right) \text {. }
$$

Each point $\left(x^{\prime}, y_{i}\right)$ can be made the center of an interval $2 \sigma_{i}$ parallel to the axis, in which the variation of $f(x, y)$ does not exceed a prescribed $\epsilon$. Let $\sigma$ be the smallest of the numbers $\sigma_{i}$ and mark out a strip of $T^{\prime}$ bounded by the parallels

$$
x=x^{\prime}-\sigma, \quad x=x^{\prime}+\sigma .
$$

Take now any two points $(x, y),\left(x^{\prime \prime}, y^{\prime \prime}\right)$ in the interior of the strip such that $\left|y-y^{\prime \prime}\right| \leqq D / 2$, and make each point the center of an interval of length $D$

* The proof given above is much simpler than that of BAIRE (loc. cit., pp. 88-94), who does not make use of the theorem of which III was an extension.

$\dagger$ The term "uniformly continuous" here signifies that if any $\varepsilon$ is arbitrarily prescribed, a value $\delta$ can be found such that for the vicinity of all points $\left(x^{\prime}, y^{\prime}\right)$ of the line considered the variation of $f(x, y)$ will be less than $\varepsilon$ when $\left|x-x^{\prime}\right| \leqq \delta,\left|y-y^{\prime}\right| \leqq \delta$, provided, of course, $(x, y)$ lies within the field $T$. 
parallel to the axis of $y$. It has been seen that the variation of $f(x, y)$ in either of these intervals does not exceed $\epsilon$. Since also they will cut in common some one of the $n$ intervals $\sigma_{i}$, we have

$$
\left|f(x, y)-f\left(x^{\prime \prime}, y^{\prime \prime}\right)\right| \leqq 3 \epsilon .
$$

If now we confine ourselves to the strip $S$ bounded by

$$
x=x^{\prime}-\frac{\sigma}{2}, \quad x=x^{\prime}+\frac{\sigma}{2},
$$

every point in $S$ may be made the center of a square, the sides of which are parallel to the axes and have a length equal to the smaller of the two numbers $\sigma$ and $D$. Within the square (or such part of the square as is contained in $T$ ) the variation of $f(x, y)$ will not exceed $6 \epsilon$.

The strip $S$ may now be substituted in place of $T$, and the reasoning be repeated. To a sequence of indefinitely diminishing values $\epsilon_{1}, \epsilon_{2}, \epsilon_{3}, \ldots$ there corresponds a series of strips of decreasing breadth, contained each in the preceding, and the limit is a line $x=\bar{x}$ on which $f(x, y)$ is uniformly continuous. Obviously, also, such lines will be found between any two parallels to the axis of $y$, and they are accordingly everywhere dense in $T$.

Suppose that

\section{§4. Theorems concerning integrals.}

$$
F(x) \equiv \int_{c}^{d} f(x, y) d y
$$

is the integral of any function $f(x, y)$ which in the rectangle $T$ is continuous in $x$ and $y$ separately. We have just found in $T$ a strip $S$ for which the following property holds: If $\left|x^{\prime}-x^{\prime \prime}\right|$ does not exceed $\sigma$, then

$$
\left|f\left(x^{\prime}, y\right)-f\left(x^{\prime \prime}, y\right)\right| \leqq 3 \epsilon \text {. }
$$

Consequently we have for the difference of the values of (15) along the parallel paths $x=x^{\prime}, x=x^{\prime \prime}$

$$
\left|F\left(x^{\prime}\right)-F\left(x^{\prime \prime}\right)\right|=\left|\int_{c}^{d} f\left(x^{\prime}, y\right) d y-\int_{c}^{d} f\left(x^{\prime \prime}, y\right) d y\right| \leqq 3 \epsilon(d-c) .
$$

In other words, in the interval $(a, b)$ may be found a subinterval in which the variation of $F(x)$ does not exceed $\zeta_{1}=6 \epsilon(d-c)$. The conclusion concerning the pointwise discontinuous character of $F(x)$ which has been stated previously in theorem VI of the introduction, follows at once in the manner explained in $\S 1$.

Consider next the difference quotient

$$
\frac{F(x+\Delta x)-F(x)}{\Delta x}=\int_{c}^{d} \frac{f(x+\Delta x, y)-f(x, y)}{\Delta x} d y
$$


If $f(x, y)$ has a derivative with respect to $x$, the application of the mean-value theorem gives for the value of the right-hand member

$$
\int_{c}^{d} f_{x}^{\prime}(x+\theta \cdot \Delta x, y) d y
$$

where $\theta$ is a number between 0 and 1 which varies with $y$. If, now, $f_{x}^{\prime}(x, y)$ is continuous in $x$ and $y$ separately, there is a strip of $T$ between two appropriately chosen parallels $x=x^{\prime} \pm \delta$, within which the variation of $f_{x}^{\prime}(x, y)$ for constant $y$ will not exceed $3 \epsilon$. Hence when $x$ and $x+\Delta x$ both lie between these limits for $x$, we have

$$
\left|\int_{c}^{d} f_{x}^{\prime}(x+\theta \cdot \Delta x, y) d y-\int_{c}^{d} f_{x}^{\prime}(x, y) d y\right| \leqq 3 \epsilon(d-c) .
$$

Consequently

where

$$
\frac{F(x+\Delta x)-F(x)}{\Delta x}=\int_{c}^{d} f_{x}^{\prime}(x, y) d y+\epsilon_{1},
$$

$$
\left|\epsilon_{1}\right| \leqq 3 \epsilon(d-c) \text {. }
$$

The interval $\left(x^{\prime}-\delta, x^{\prime}+\delta\right)$ and the corresponding strip of $T$ may now be substituted in place of $(a, b)$ and the field $T$, and the argument may be repeated with a smaller value of $\epsilon$. Continuing in this way we obtain with diminishing $\epsilon$ a series of intervals contained each in the interior of the preceding and having for their limit a point $x$ at which

$$
\lim _{\Delta x=0} \frac{F(x+\Delta x)-F(x)}{\Delta x}=F^{\prime}(x)=\int_{c}^{d} f_{x}^{\prime}(x, y) d y .
$$

As the argument applies to any portion of $(a, b)$, the first part of theorem VII is established.

The second part of the theorem follows from the remark that in the succession of strips diminishing with $\epsilon$ the variation of $f_{x}^{\prime}(x, y)$ for constant $y$ does not exceed $3 \epsilon$, so that the variation of

$$
F_{1}(x)=\int_{c}^{d} f_{x}^{\prime}(x, y) d x
$$

does not exceed $3 \epsilon(d-c)$. The limiting point $x$ is therefore a point of continuity of $F_{1}(x)$.

Let

$$
\text { §. On series. }
$$

$$
S(x)=u_{1}(x)+u_{2}(x)+\cdots
$$

be a series, whose terms are continuous in $(a, b)$ and which converges at every point of this interval. Denote by $S_{n}(x)$ the sum of the first $n$ terms of the 
series. To each point $x_{i}$ of the interval a number $n_{i}$ can be so assigned that

$$
\left|S_{n_{i}+m}\left(x_{i}\right)-S\left(x_{i}\right)\right|<\epsilon \quad(m=1,2,3, \cdots) .
$$

If $\epsilon$ is fixed and a sufficiently large $N$ is selected, the set of points $x=q_{i}$ for which $n_{i}<N$ will be everywhere dense in some subinterval, $\left(a^{\prime}, b^{\prime}\right)$. Let $Q$ denote this set of points. Contract this interval so much as may be necessary to make the variation of the continuous function $S_{N}(x)$ within $\left(a^{\prime}, b^{\prime}\right)$ less than $\epsilon$. Then for and $q_{2}$, any two points of $Q$, we have

and

$$
\begin{aligned}
& \left|S_{N}\left(q_{1}\right)-S\left(q_{1}\right)\right|<\epsilon, \\
& \left|S_{N}\left(q_{2}\right)-S\left(q_{2}\right)\right|<\epsilon,
\end{aligned}
$$

$$
\left|S_{N}\left(q_{1}\right)-S_{N}\left(q_{2}\right)\right|<\epsilon
$$

Hence

$$
\left|S\left(q_{1}\right)-S\left(q_{2}\right)\right|<3 \epsilon ;
$$

that is to say, the variation of $S(x)$ in $\left(a^{\prime}, b^{\prime}\right)$ over the set $Q$ does not exceed $6 \epsilon$. Consider next a point $p$ in $\left(a^{\prime}, b^{\prime}\right)$ which does not belong to $Q$. A fixed value $N^{\prime} \geqq N$ can be found such that

$$
\left|S_{N^{\prime}}(p)-S(p)\right|<\epsilon^{\prime} .
$$

As the points $q_{i}$ are everywhere dense in $\left(a^{\prime} b^{\prime}\right)$, one of these points be found so near to $p$ that

Since also

$$
\left|S_{N^{\prime}}(p)-S_{N^{\prime}}\left(q_{i}\right)\right|<\epsilon^{\prime} \text {. }
$$

$$
\left|S_{N^{\prime}}\left(q_{i}\right)-S\left(q_{i}\right)\right|<\epsilon,
$$

it follows from the last three inequalities that

$$
\left|S(p)-S\left(q_{i}\right)\right|<\epsilon+2 \epsilon^{\prime} .
$$

But $\epsilon^{\prime}$ is arbitrary. We conclude therefore from (16) and (17) that the variation of $S(x)$ within $\left(a^{\prime}, b^{\prime}\right)$ can not exceed $\zeta_{1}=8 \epsilon$. By $\S 2$ this gives at once the following theorem :

Theorem VIII. If the series

$$
S(x)=u_{1}(x)+u_{2}(x)+u_{3}(x)+\cdots
$$

converges at every point of an interval $(a, b)$ and each term of the series is a continuous function of $x$, the sum of the series can be at most only pointwise discontinuous in ( $a, b)$ (BAIRE).*

This theorem was first proved by OsGood $\nmid$ for the case in which the limit is a

* Loc. cit., p. 62, or Bericht, p. 224.

†American Journal of Mathematios, vol. 19 (1897). 
continuous function, and he showed, moreover, that the points of uniform convergence* are everywhere dense. Recently Hobson $\dagger$ has proved that the points of uniform convergence are also dense in the more comprehensive case considered in theorem VIII. This conclusion results also from the proof given above, for it can be shown at once that all the points of continuity for $S(x)$ obtained through the demonstration are points of uniform convergence. Take, in fact, any one of these points. It can be made first the center of an interval $(\alpha, \beta)$ in which the variation of $S(x)$ is less than $\epsilon$. But it also lies in an interval $\left(a^{\prime}, b^{\prime}\right)$ in which the points $q_{i}$ are everywhere dense. For any of these points we had

$$
\left|S_{n}\left(q_{i}\right)-S\left(q_{i}\right)\right|<\epsilon
$$$$
(n \geqq N) \text {. }
$$

In the vicinity of any point $p$ of $\left(a^{\prime}, b^{\prime}\right)$ we can find a point $q_{i}$ so near that, for given $n$ and $\epsilon^{\prime}$,

$$
\left|S_{n}(p)-S_{n}\left(q_{i}\right)\right|<\epsilon^{\prime} .
$$

But for two points $q_{i}$ and $p$ interior to $(\alpha, \beta)$,

$$
\left|S\left(q_{i}\right)-S(p)\right|<\epsilon .
$$

Hence from the last three inequalities

$$
\left|S_{n}(p)-S(p)\right| \leqq 2 \epsilon
$$$$
(n>N) \text {. }
$$

The two inequalities (18), (19) hold simultaneously for the common portion of $\left(a^{\prime}, b^{\prime}\right)$ and $(\alpha, \beta)$, and consequently the center of $(\alpha, \beta)$ is, as stated, a point of uniform convergence.

\footnotetext{
* By a point of uniform convergence is to be understood one which, for any arbitrarily assigned $\varepsilon$, can be enclosed in an interval in which$$
\left|S(x)-S_{n}(x)\right|<\varepsilon
$$

for all values of $x$ and for a sufficiently large $N$.

† Proceedings of the London Mathematical Society, vol. 34 (1902), p. 245, or Acta Mathematioa, vol. 27 (1903), p. 209. An earlier proof was given in Townsend's dissertation (Göttingen, 1900), p. 68.
} 